\title{
Adaptive Equalizer Design for Unmanned Aircraft Vehicle Image Transmission over Relay Channels
}

\author{
Huang Wenqian (D) and Ding Wenrui \\ School of Electronic and Information Engineering, Beihang University, Beijing, China \\ Correspondence should be addressed to Huang Wenqian; huangwenqian@buaa.edu.cn
}

Received 8 December 2017; Revised 23 February 2018; Accepted 5 March 2018; Published 11 April 2018

Academic Editor: Hongwei Wang

Copyright (c) 2018 Huang Wenqian and Ding Wenrui. This is an open access article distributed under the Creative Commons Attribution License, which permits unrestricted use, distribution, and reproduction in any medium, provided the original work is properly cited.

\begin{abstract}
A novel length adaptive method is proposed for time domain equalizer by taking the channel attenuation ratio between different multipath components into account in UAV-UAV and UAV-ground channels. Then, considering received image quality, the minimum bit error ratio (MBER) criterion is exploited to design adaptive equalizers for both amplify-and-forward (AF) and decode-and-forward (DF) relaying systems by the proposed length adaptive method. Results show that proposed MBER adaptive equalizers outperform the traditional ones in both AF relaying and DF relaying as channel attenuation ratio in UAV-ground channel increases. Moreover, DF outperforms AF as channel attenuation ratio in UAV-UAV channel increases. Furthermore, bit error ratio (BER) and peak signal-to-noise ratio (PSNR) performances in both AF and DF are evaluated to show the enhancement by the proposed MBER adaptive equalizers.
\end{abstract}

\section{Introduction}

With the explosive growth of using unmanned aircraft vehicle (UAV) for various applications, there is a tremendous demand for image wireless transmission in UAV remote sensing system $[1,2]$. The performance of traditional direct UAV-to-ground communication is severely degraded by the fading of the wireless channel and can be easily shadowed by buildings in urban areas or by mountains in rural areas, leading to drop in the quality of the received images. Thus it is imperative to develop the future communication schemes to mitigate the impact caused by the fading and shadowing.

Relaying as an approach to increase the reliability and extend the coverage area of the UAV remote sensing system, as depicted in Figure 1, has attracted a huge interest [3-5]. In [3], UAVs are deployed as flying relays between aerial and ground mesh networks in an emergency scenario. Reference [5] investigated how to deploy UAVs as flying relays to increase coverage in the wireless network. Generally, there are mainly two widely used relaying schemes: amplify-andforward (AF) and decode-and-forward (DF). In AF, the relay amplifies and retransmits the received signal from the source to the destination. In DF, the relay decodes the signal and then forwards it to the destination.

According to recent measurement campaign and literatures [6-8], UAV-to-ground and UAV-to-UAV channels, two kinds of channels widely used in UAV-based relaying systems, can be typically modeled as frequency-selective multipath channels whose dispersive nature can easily cause the intersymbol interference (ISI), leading to inevitable performance degradation. Additionally, channel taps of UAV-ground channel are larger than those of UAV-UAV channel [9-11]. Reference [7] found that the UAV-ground channel in over-sea scenarios can be well modeled by the two-ray model plus an intermittent multipath component. UAV-ground channels in hilly/mountainous and suburban/near-urban scenarios can be modeled by nine-tap multipath channels [8, 11]: line-ofsight (LoS) component as the first tap, Ground Reflection (GR) component as the second tap, and seven intermittent components.

High-speed single-carrier wideband transmission systems are widely used in aeronautical communication due to severe size, weight, and power (SWAP) of drones [12], and ISI caused by multipath propagation tends to be the main 


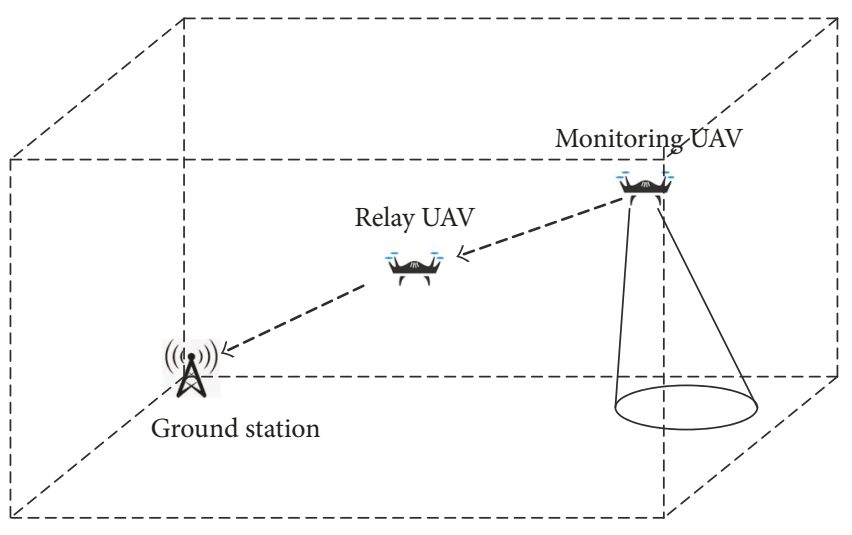

FIgURE 1: UAV remote sensing system.

factor limiting the reliability of these systems. Thus, time domain equalizer plays an important role mitigating ISI in aeronautical communication [12]. Zero-forcing (ZF) and minimum mean-squared error (MMSE) equalizers are proposed for single-hop aeronautical communication in [13].

In traditional terrestrial relaying systems, [14] designed the minimum bit error ratio (MBER) equalizer for AF relaying. A new detector for AF relaying is proposed in [15] using a minimum symbol error ratio (MSER) equalizer. MMSE and MBER equalizers are proposed and investigated in [16] for nonorthogonal AF relaying.

Different from terrestrial relaying systems, LoS communication links can always be established in UAV-based relaying systems. In particular, UAV-ground channel has some unique features due to the GR multipath component which can more easily cause severe ISI [9]. To the best of our knowledge, there is lack of works dealing with equalizer design for UAV-based relaying systems that take the characteristics of UAV-UAV and UAV-ground channels into account in previous literatures.

The main contributions of this work are the following:

(i) A length adaptive method for equalizer design is proposed according to channel attenuation ratio between different multipath components.

(ii) For image wireless transmission in UAV-based relaying systems, MBER criterion is exploited to design equalizers in both AF and DF relaying systems, since MBER, rather than the widely used MMSE, is more suitable to reflect image quality with high fidelity $[17,18]$.

(iii) Bit error ratio (BER) and peak signal-to-noise ratio (PSNR) performances of AF and DF relaying with MBER adaptive equalizers are evaluated in relation to critical system parameters like the number of training symbols, defined complexity penalty parameter of DF, and average signal-to-noise ratio (SNR), which are important for practical application in UAV remote sensing system.

Notation. Bold uppercase and lowercase letters denote matrices and vectors, respectively. The superscript $(\cdot)^{H}$ denotes the conjugate transpose of a matrix. $\operatorname{Re}(\cdot)$ represents the real part of a complex number. $\operatorname{sgn}(\cdot)$ denotes the sign function.

\section{System Model}

As depicted in Figure 2, we consider a three-node relaying system consisting of a monitoring UAV (M-UAV) as the source node, a ground station (GS) as the destination node, and a relay UAV (R-UAV) as the relay node without direct path between M-UAV and GS (e.g., due to shadowing or large separation). Each node is equipped with only one antenna and operates in the half-duplex mode. Two UAVs are assumed to have the same height $h_{U}$. Thus, the three-dimensional (3D) coordinates of M-UAV, R-UAV, and GS can be denoted as $\mathbf{s}=\left(x_{s}, y_{s}, h_{U}\right), \mathbf{r}=\left(x_{r}, y_{r}, h_{U}\right)$, and $\mathbf{d}=\left(x_{d}, y_{d}\right.$, $\left.h_{G}\right)$, respectively.

The image bit stream is sent to GS from M-UAV via R-UAV using AF or DF relaying. In AF relaying, M-UAV transmits image bit stream to R-UAV in the first time slot and then R-UAV amplifies and retransmits the received bit stream to the GS in the second time slot. In DF relaying, MUAV transmits the image bit stream to R-UAV in the first time slot and R-UAV decodes and retransmits the image bit stream in the second time slot. In AF relaying, the equalizer is considered only at GS; in DF relaying, two equalizers are considered at both R-UAV and GS.

2.1. UAV-UAV Channel Model. Considering UAV-UAV channel as a discrete-time Rician multipath channel, the channel outputs at R-UAV for both AF and DF can be expressed as

$$
r_{k}^{A}=r_{k}^{D}=h_{0}^{U U} x_{k}+h_{1}^{U U} x_{k-1}+n .
$$

$x_{k} \in\{ \pm 1\}$ is $k$ th BPSK data symbol, which is coded and modulated from image bit stream. $n$ denotes additive white Gaussian noise (AWGN). $h_{0}^{U U}$ and $h_{1}^{U U}$ denote the channel attenuation of LoS and scattered components, respectively. The channel attenuation of LoS component in $\mathrm{dB}$ can be expressed as [6]

$$
h_{0}^{U U}=10 \alpha \log \left(\frac{4 \pi f_{c} R_{\mathrm{LoS}}}{c}\right)+\psi_{\mathrm{LoS}}
$$

where $\alpha$ is path loss exponent, $f_{c}$ is the carrier frequency, $c$ is the speed of light, and $R_{\mathrm{LoS}}$ denotes the range of LoS component. $\psi_{\mathrm{LoS}} \sim N\left(\mu_{\mathrm{LoS}}, \delta_{\mathrm{LoS}}\right)$ is the shadow fading with normal distribution in $\mathrm{dB}$ for LoS link. The channel attenuation of scattered components in $\mathrm{dB}$ can be expressed as

$$
h_{1}^{U U}=h_{0}^{U U}-K
$$

where $K$ in $\mathrm{dB}$ is the Rician $\mathrm{K}$-factor for UAV-UAV channel.

2.2. UAV-Ground Channel Model. Considering the discretetime UAV-ground multipath channel based on the recent 

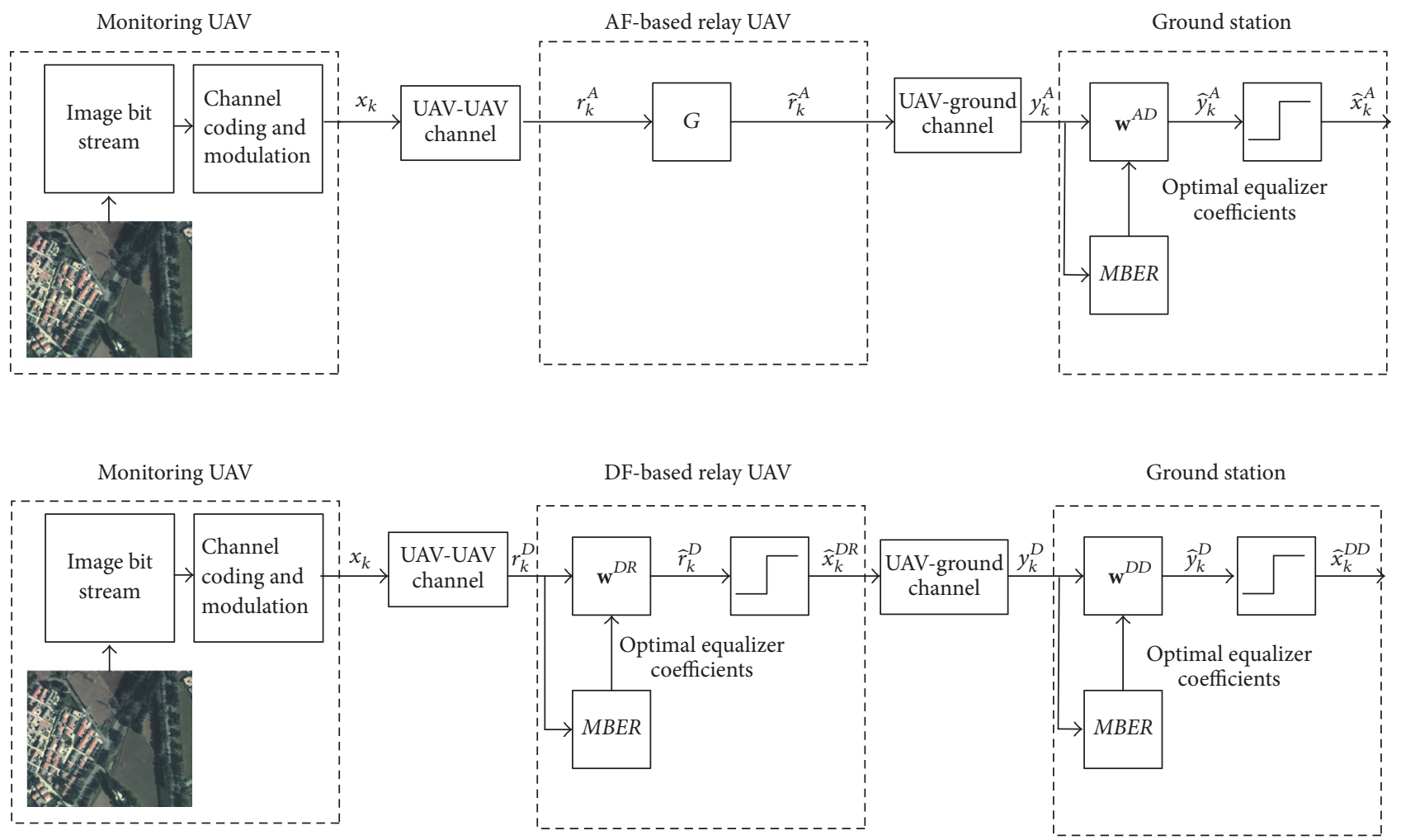

Figure 2: System model.

works $[7,8,11]$, the channel outputs at GSs for AF and DF can be expressed as

$$
\begin{aligned}
& y_{k}^{A}=\sum_{i=0}^{C_{U G}} h_{i}^{U G} \widehat{r}_{k-i}^{A}+n, \\
& y_{k}^{D}=\sum_{i=0}^{C_{U G}} h_{i}^{U G} \widehat{x}_{k-i}^{D R}+n,
\end{aligned}
$$

respectively. $C_{U G} \geq 3$ denotes the channel taps for UAVground channel. The value of these channel taps depends on environment; for instance, $C_{U G}=3$ in the over-water scenarios, while $C_{U G}=9$ in the mountainous and hilly scenarios [7, 11]. $h_{0}^{U G}$ denotes the channel attenuation of LoS component and can be expressed the same as (2):

$$
h_{0}^{U G}=10 \alpha \log \left(\frac{4 \pi f_{c} R_{\mathrm{LoS}}}{c}\right)+\psi_{\mathrm{LoS}}
$$

Similarly, $h_{1}^{U G}$ denotes the channel attenuation of the GR component and can be expressed as

$$
h_{1}^{U G}=10 \alpha \log \left(\frac{4 \pi f_{c} R_{\mathrm{GR}}}{c}\right)+\psi_{\mathrm{GR}}
$$

where $\psi_{\mathrm{GR}} \sim N\left(\mu_{\mathrm{GR}}, \delta_{\mathrm{GR}}\right)$ is the shadow fading with normal distribution in $\mathrm{dB}$ for $\mathrm{GR}$ link and $R_{\mathrm{GR}}$ denotes the range of GR component.
Assuming a UAV-ground channel geometry as depicted in Figure $3[10,19]$, the ranges of LoS and GR components in UAV-ground channel can be calculated as

$$
\begin{aligned}
& R_{\mathrm{LoS}}=\sqrt{\left(h_{U}-h_{G}\right)^{2}+d^{2}}, \\
& R_{\mathrm{GR}}=\sqrt{\left(h_{U}+h_{G}\right)^{2}+d^{2}},
\end{aligned}
$$

where $d$ denotes the distance between R-UAV and GS in the horizontal plane.

For simplicity, the channels between all nodes are assumed to be constant during the transmission time of one image (moving distances of nodes can be negligible within this period, since UAV datalink speed, whose carrier frequency usually uses L-band (960-997 MHz) or C-band (5030$5091 \mathrm{MHz}$ ) [9], can reach $10 \mathrm{Mbps}$ [20]).

\section{MBER Adaptive Equalizer Design for Relaying System}

3.1. Length Adaptive Mechanism. For example, in (1), the desired received signal component for $k$ th symbol is $h_{0}^{U U} x_{k}$, but the component $h_{1}^{U U} x_{k-1}$, also called ISI, could have an influence on the received signal decision performance due to the delay transmission of $(k-1)$ th symbol $x_{k}$ over the channel $h_{1}^{U U}$. This decision performance depends on some system parameters. One of them is the channel attenuation ratio between LoS and GR components in UAV-ground channel or scattered components in UAV-UAV channel. For 


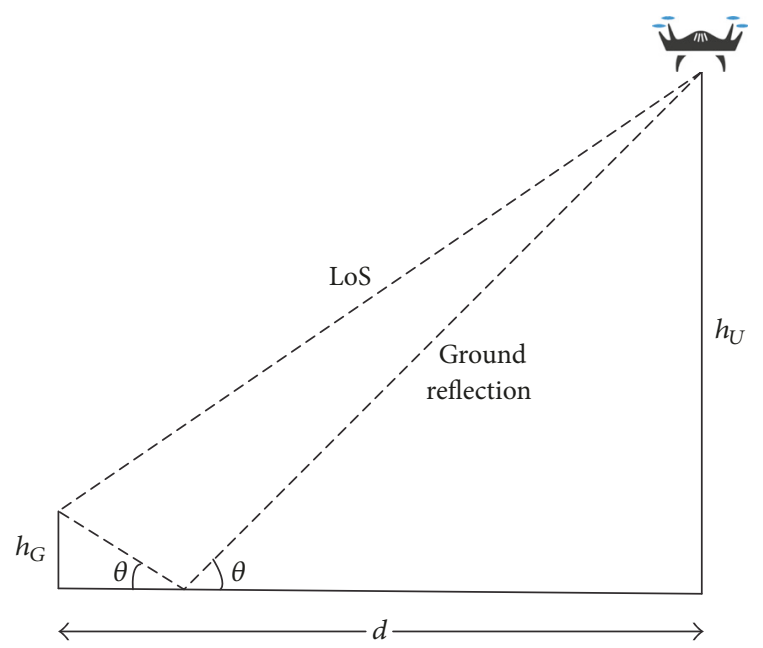

FIGURE 3: UAV-ground channel geometry.

example, in (1), if the magnitude of component $h_{0}^{U U} x_{k}$ is much larger than that of component $h_{1}^{U U} x_{k-1}$, the ISI influence on decision performance is very limited; otherwise, the decision performance could be severely degraded.

Generally, the reliability performance of equalizers aiming at mitigating ISI increases with the equalizer length but at the cost of more training time. Considering equalizer performance as well as training time, traditional fixed length equalizers have limited adaptive performance: equalizers with large length could generate severe signal processing delay due to more training time, while the one with small length could not mitigate ISI as much as possible.

Thus, the proposed length adaptive mechanism is that equalizer can adjust its length to optimal ISI mitigation performance according to the channel attenuation ratio between different multipath components:

$$
\frac{L-L_{\min }}{L_{\max }-L_{\min }}=\frac{\gamma-\gamma_{\min }}{\gamma_{\max }-\gamma_{\min }},
$$

where $L$ denotes the equalizer length using proposed method. $L_{\min }=2$ is the minimal equalizer length. $L_{\max }$ is the maximal equalizer length depending on the calculating resources of receivers.

In UAV-ground channel, the channel attenuation ratio in $\mathrm{dB}$ between LoS and GR components can be expressed as

$$
\gamma^{U G}=h_{1}^{U G}-h_{0}^{U G} \text {. }
$$

We assume that the position of R-UAV is known by GS by control signals at first; then $\gamma^{U G}$ can be calculated using (6) and (7) at GS. Thus the length of equalizer at GS can be adjusted using (9).

In UAV-UAV channel, this ratio is also related to Rician K-factor:

$$
\gamma^{U U}=\frac{1}{K} .
$$

$\gamma_{\min }$ and $\gamma_{\max }$ denote the maximal and minimal channel attenuation ratio, respectively. The values of $\gamma_{\min }$ and $\gamma_{\max }$ depend on the environment $[7,8,11]$.
The proposed optimal equalizer length can be rewritten as

$$
L=f(\gamma)=\frac{\gamma-\gamma_{\min }}{\gamma_{\max }-\gamma_{\min }}\left(L_{\max }-L_{\min }\right)+L_{\min } .
$$

3.2. Equalizer Design for AF. The equalizer at GS in AF relaying can be described by the vector $\mathbf{w}^{A D}=\left\{w_{1}^{A D} \cdots w_{L^{A}}^{A D}\right\}^{T}$ and equalizer outputs can be expressed as

$$
\widehat{y}_{k}^{A}=\sum_{i=1}^{L^{A}} w_{i}^{A D} \widehat{r}_{k-i}^{A}+n,
$$

where $L^{A}$ denotes the optimal length of equalizer at GS in AF relaying and can be calculated by

$$
L^{A}=f\left(\gamma^{U G}\right) .
$$

The equalizer outputs are then passed to the decision device and the decision can be expressed as

$$
\widehat{x}_{k}^{A}=\operatorname{sgn}\left(\operatorname{Re}\left(\hat{y}_{k}^{A}\right)\right)= \begin{cases}+1 & \operatorname{Re}\left(\hat{y}_{k}^{A}\right) \geq 0, \\ -1 & \operatorname{Re}\left(\hat{y}_{k}^{A}\right) \leq 0 .\end{cases}
$$

Then, based on (1), (4), (13), and (15), the end-to-end signal transmission over the whole link can be expressed as

$$
\begin{aligned}
\hat{x}_{k}^{A} & =\operatorname{sgn}\left(\operatorname{Re}\left(\hat{y}_{k}^{A}\right)\right) \\
& =\operatorname{sgn}\left(\operatorname{Re}\left(\left(\mathbf{w}^{A D}\right)^{H} \mathbf{H}^{A 2} G \mathbf{H}^{A 1} \mathbf{x}_{k}^{A}+n\right)\right)
\end{aligned}
$$

where $\mathbf{x}_{k}^{A}=\left\{x_{k}, x_{k-1}, \ldots, x_{k-\left(L^{A}+2+C_{U G}-1\right)}\right\}^{T}$ is a vector of channel inputs from M-UAV. $G=\sqrt{1 /\left(\left(h_{0}^{U U}\right)^{2}+\left(h_{1}^{U U}\right)^{2}\right)}$ is ideal amplifying gain at R-UAV for each received symbol. $\mathbf{H}^{A 2}$ is the $L^{A} \times\left(L^{A}+C_{U G}\right)$ Toeplitz convolution matrix; $\mathbf{H}^{A 1}$ is the $\left(L^{A}+C_{U G}\right) \times\left(L^{A}+C_{U G}+2\right)$ Toeplitz convolution matrix. $\mathbf{H}^{A 2}$ and $\mathbf{H}^{A 1}$ can be expressed as

$$
\begin{aligned}
\mathbf{H}^{A 2} & =\left[\begin{array}{ccccccc}
h_{0}^{U G} & h_{1}^{U G} & \cdots & h_{C_{U G}^{U G}} & 0 & \cdots & 0 \\
0 & h_{0}^{U G} & h_{1}^{U G} & \cdots & h_{C_{U G}^{U G}} & 0 & \vdots \\
& & & & & \ddots & \\
0 & \cdots & 0 & h_{0}^{U G} & h_{1}^{U G} & \cdots & h_{C_{U G}^{U G}}^{U G}
\end{array}\right], \\
\mathbf{H}^{A 1} & =\left[\begin{array}{ccccc}
h_{0}^{U U} & h_{1}^{U U} & 0 & \cdots & 0 \\
0 & h_{0}^{U U} & h_{1}^{U U} & 0 & \vdots \\
& & & \ddots & \\
0 & \cdots & 0 & h_{0}^{U U} & h_{1}^{U U}
\end{array}\right] .
\end{aligned}
$$
fined as

The BER for AF relaying after equalizer $\mathbf{w}^{A D}$ can be de-

$$
\begin{aligned}
P_{E}^{A} & =\operatorname{Pr}\left(\hat{x}_{k}^{A} x_{k}<0\right) \\
& =\int_{-\infty}^{0} p\left(\mathbf{w}^{A D}, \operatorname{Re}\left(\hat{y}_{k}^{A}\right)\right) x_{k} d\left(\operatorname{Re}\left(\hat{y}_{k}^{A}\right)\right),
\end{aligned}
$$


where $p\left(\mathbf{w}^{A D}, \operatorname{Re}\left(\widehat{y}_{k}^{A}\right)\right)$ denotes the probability density function (PDF) of $\operatorname{Re}\left(\hat{y}_{k}^{A}\right)$ and is also the function of $\mathbf{w}^{A D}$. Then, the tap coefficient vector for MBER adaptive equalizer at GS for AF relaying can be expressed as

$$
\mathbf{w}^{A D}=\arg \min _{w} P_{E}^{A}\left(\mathbf{w}^{A D}\right) .
$$

3.3. Equalizer Design for DF. Equalizers at R-UAV and GS can be described by the vectors $\mathbf{w}^{D R}=\left\{w_{1}^{D R} \cdots w_{L^{D R}}^{D R}\right\}^{T}$ and $\mathbf{w}^{D D}=\left\{w_{1}^{D D} \cdots w_{L^{D D}}^{D D}\right\}^{T}$, respectively. Then, the equalizer outputs at R-UAV and GS can be expressed as

$$
\begin{aligned}
& \widehat{r}_{k}^{D}=\sum_{i=1}^{L^{D R}} w_{i}^{D R} r_{k-i}^{D}+n, \\
& \widehat{y}_{k}^{D}=\sum_{i=1}^{L^{D D}} w_{i}^{D D} y_{k-i}^{D}+n,
\end{aligned}
$$

respectively. $L^{D R}$ and $L^{D D}$ denote the optimal equalizer lengths. Similar to (14), these two lengths can be calculated by

$$
\begin{aligned}
& L^{D R}=f\left(\gamma^{U U}\right), \\
& L^{D D}=f\left(\gamma^{U G}\right) .
\end{aligned}
$$

The two equalizer outputs are then passed to the decision devices and the decisions can be expressed as

$$
\begin{aligned}
& \widehat{x}_{k}^{D R}=\operatorname{sgn}\left(\operatorname{Re}\left(\widehat{r}_{k}^{D}\right)\right)= \begin{cases}+1 & \operatorname{Re}\left(\widehat{r}_{k}^{D}\right) \geq 0, \\
-1 & \operatorname{Re}\left(\widehat{r}_{k}^{D}\right) \leq 0,\end{cases} \\
& \widehat{x}_{k}^{D D}=\operatorname{sgn}\left(\operatorname{Re}\left(\hat{y}_{k}^{D}\right)\right)= \begin{cases}+1 & \operatorname{Re}\left(\widehat{y}_{k}^{D}\right) \geq 0, \\
-1 & \operatorname{Re}\left(\hat{y}_{k}^{D}\right) \leq 0 .\end{cases}
\end{aligned}
$$

Similar to the equalizer design in AF, the end-to-end signal transmission at R-UAV and GS in DF can be rewritten as

$$
\begin{aligned}
& {\widehat{x}_{k}^{D R}}^{D R}=\operatorname{sgn}\left(\operatorname{Re}\left(\left(\mathbf{w}^{D R}\right)^{H} \mathbf{H}^{D 1} \mathbf{x}_{k}+n\right)\right), \\
& \widehat{x}_{k}^{D D}=\operatorname{sgn}\left(\operatorname{Re}\left(\left(\mathbf{w}^{D D}\right)^{H} \mathbf{H}^{D 2} \widehat{\mathbf{x}}_{k}^{D R}+n\right)\right),
\end{aligned}
$$

respectively. $\mathbf{x}_{k}=\left\{x_{k}, x_{k-1}, \ldots, x_{k-\left(L^{D R}+2-1\right)}\right\}^{T}$ and $\widehat{\mathbf{x}}_{k}^{D R}=$ $\left\{\widehat{x}_{k}^{D D}, \widehat{x}_{k-1}^{D D}, \ldots, \widehat{x}_{k-\left(L^{D D}+C_{U G}-1\right)}^{D D}\right\}^{T}$ are two vectors of channel inputs from M-UAV and R-UAV. $\mathbf{H}^{D 2}$ and $\mathbf{H}^{D 1}$ are $L^{D R} \times$ $\left(L^{D R}+C_{U G}\right)$ and $L^{D D} \times\left(L^{D D}+2\right)$ Toeplitz convolution matrices, respectively. These two matrices can be expressed as

$$
\begin{aligned}
\mathbf{H}^{D 2} & =\left[\begin{array}{ccccccc}
h_{0}^{U G} & h_{1}^{U G} & \cdots & h_{C_{U G}^{R G}}^{R D} & 0 & \cdots & 0 \\
0 & h_{0}^{U G} & h_{1}^{U G} & \cdots & h_{C_{U G}^{U G}} & 0 & \vdots \\
& & & & & \ddots & \\
0 & \cdots & 0 & h_{0}^{U G} & h_{1}^{U G} & \cdots & h_{C_{U G}^{U G}}^{U G}
\end{array}\right], \\
\mathbf{H}^{D 1} & =\left[\begin{array}{ccccc}
h_{0}^{U U} & h_{1}^{U U} & 0 & \cdots & 0 \\
0 & h_{0}^{U U} & h_{1}^{U U} & 0 & \vdots \\
& & & \ddots & \\
0 & \cdots & 0 & h_{0}^{U U} & h_{1}^{U U}
\end{array}\right] .
\end{aligned}
$$

Following the same approach as carried out to obtain (19), the optimal tap coefficient vectors for MBER adaptive equalizers at R-UAV and GS for DF relaying can be expressed as

$$
\begin{aligned}
& \mathbf{w}^{D R}=\arg \min _{\mathbf{w}} P_{E}^{D R}\left(\mathbf{w}^{D R}\right), \\
& \mathbf{w}^{D D}=\arg \min _{\mathbf{w}} P_{E}^{D D}\left(\mathbf{w}^{D D}\right),
\end{aligned}
$$

where $P_{E}^{D R}\left(\mathbf{w}^{D D}\right)$ and $P_{E}^{D D}\left(\mathbf{w}^{D R}\right)$ can be expressed as

$$
\begin{aligned}
P_{E}^{D R} & =\operatorname{Pr}\left(\operatorname{sgn}\left(\operatorname{Re}\left(\widehat{r}_{k}^{D}\right)\right) x_{k}<0\right) \\
& =\int_{-\infty}^{0} p\left(\mathbf{w}^{D R}, \operatorname{Re}\left(\hat{r}_{k}^{D}\right)\right) x_{k} d\left(\operatorname{Re}\left(\hat{r}_{k}^{D}\right)\right), \\
P_{E}^{D D} & =\operatorname{Pr}\left(\operatorname{sgn}\left(\operatorname{Re}\left(\hat{y}_{k}^{D}\right)\right) x_{k}<0\right) \\
& =\int_{-\infty}^{0} p\left(\mathbf{w}^{D R}, \operatorname{Re}\left(\hat{y}_{k}^{D}\right)\right) x_{k} d\left(\operatorname{Re}\left(\hat{y}_{k}^{D}\right)\right) .
\end{aligned}
$$

3.4. Adaptive Equalizer Algorithm. We first consider the optimal equalizer coefficients for AF. To obtain optimal equalizer coefficients $\mathbf{w}^{A D}$, the $\operatorname{PDF}$ of $\operatorname{Re}\left(\hat{y}_{k}^{A}\right)$ is necessary. It is seen that PDF of $\operatorname{Re}\left(\hat{y}_{k}^{A}\right)$ is Gaussian mixture and can be estimated using nonparametric estimation [21]. Parzen window method is good at estimating PDF with relatively short data, which is efficient for equalizer training [21].

Given the training symbols $\left\{x_{k}, \mathbf{y}_{k}^{A}\right\}_{k=1}^{N}$ and using Parzen window function method, the $\operatorname{PDF}$ of $\operatorname{Re}\left(\widehat{y}_{k}^{A}\right)$ can be expressed as

$$
p(t)=\frac{1}{N \sqrt{2 \pi} \rho_{n}} \sum_{k=1}^{N} e^{-\left|t-\operatorname{Re}\left(\hat{y}_{k}^{A}\right)\right|^{2} / 2 \rho_{n}^{2}}
$$

where $N$ is the length of training symbols and $\rho_{n}$ is the window width. Substituting (31) in (18), the BER for AF relaying can be rewritten as

$$
P_{E}^{A}=\frac{1}{N} \sum_{k=1}^{N} Q\left(\frac{\operatorname{sgn}\left(x_{k}\right) \operatorname{Re}\left(\left(\mathbf{w}^{A D}\right)^{H} \mathbf{y}_{k}^{A}+n\right)}{\rho_{n}}\right),
$$


where $Q$ is the Gaussian error function. Then the gradient of $P_{E}^{A}$ can be given as

$$
\begin{aligned}
\nabla P_{E}^{A}= & -\frac{1}{2 N \sqrt{2 \pi} \rho_{n}} \\
& \times \sum_{k=1}^{N} e^{-\left|\operatorname{Re}\left(\mathbf{w}^{A D}\right)^{H} \mathbf{y}_{k}^{A}\right|^{2} / 2 \rho_{n}^{2}} \operatorname{sgn}\left(x_{k}\right) \mathbf{y}_{k}^{A} .
\end{aligned}
$$

Then, the solution to optimize equalizer coefficients $\mathbf{w}^{A D}$ is described as

$$
\begin{aligned}
\hat{y}_{k}^{A} & =\left(\mathbf{w}_{k-1}^{A D}\right)^{H} \mathbf{y}_{k}^{A}, \\
\mathbf{w}_{k}^{A D} & =\mathbf{w}_{k-1}^{A D}+\mu \frac{\operatorname{sgn}\left(x_{k}\right)}{2 \sqrt{2 \pi} \rho_{n}} e^{-\left|\operatorname{Re}\left(\mathbf{w}^{A D}\right)^{H} \mathbf{y}_{k}^{A}\right|^{2} / 2 \rho_{n}^{2}} \mathbf{y}_{k}^{A},
\end{aligned}
$$

where $\mu$ is the step size that has to be set to balance the convergence rate and the steady-state BER.

Following the same approach as carried out to solve (19), given the training symbols $\left\{x_{k}, \mathbf{r}_{k}^{D}, \mathbf{y}_{k}^{D}\right\}_{k=1}^{N}$ for relay and destination, respectively, and using Parzen window function method, the BER for DF relaying at relay and destination can be given as

$$
\begin{aligned}
& P_{E}^{D R}=\frac{1}{N} \sum_{k=1}^{N} Q\left(\frac{\operatorname{sgn}\left(x_{k}\right) \operatorname{Re}\left(\left(\mathbf{w}^{D R}\right)^{H} \mathbf{r}_{k}^{D}+n\right)}{\rho_{n}}\right), \\
& P_{E}^{D D}=\frac{1}{N} \sum_{k=1}^{N} Q\left(\frac{\operatorname{sgn}\left(x_{k}\right) \operatorname{Re}\left(\left(\mathbf{w}^{D D}\right)^{H} \mathbf{y}_{k}^{D}+n\right)}{\rho_{n}}\right),
\end{aligned}
$$

respectively. Then, the solution to optimize equalizer coefficients $\mathbf{w}^{D R}$ and $\mathbf{w}^{D D}$ for DF relaying at relay and destination can be described as

$$
\begin{aligned}
\widehat{r}_{k}^{D} & =\left(\mathbf{w}_{k-1}^{D R}\right)^{H} \mathbf{r}_{k}^{D}, \\
\mathbf{w}_{k}^{D R} & =\mathbf{w}_{k-1}^{D R}+\mu \frac{\operatorname{sgn}(x)}{2 \sqrt{2 \pi} \rho_{n}} e^{-\left|\operatorname{Re}\left(\mathbf{w}^{D R}\right)^{H} \mathbf{r}_{k}^{D}\right|^{2} / 2 \rho_{n}^{2}} \mathbf{r}_{k}^{D}, \\
\widehat{y}_{k}^{D} & =\left(\mathbf{w}_{k-1}^{D D}\right)^{H} \mathbf{y}_{k}^{D}, \\
\mathbf{w}_{k}^{D D} & =\mathbf{w}_{k-1}^{D D}+\mu \frac{\operatorname{sgn}\left(x_{k}\right)}{2 \sqrt{2 \pi} \rho_{n}} e^{-\left|\operatorname{Re}\left(\mathbf{w}^{D D}\right)^{H} \mathbf{y}_{k}^{D}\right|^{2} / 2 \rho_{n}^{2}} \mathbf{y}_{k}^{D} .
\end{aligned}
$$

3.5. Performance Comparison. The end-to-end BER ratio of $\mathrm{AF}$ and DF with MBER adaptive equalizers can be defined as

$$
\eta=\frac{P_{E}^{A}}{\alpha P_{E}^{D}},
$$

where $\alpha$ denotes the complexity penalty parameter of $\mathrm{DF}$, which represents the complexity of DF relaying technology. On one hand, DF relaying has more complexity in signal processing, leading to more delay and resource consumption
TABLE 1: Simulation parameters.

\begin{tabular}{lc}
\hline Parameter & Value \\
\hline$\rho_{n}$ & $4 \sigma_{n}^{2}[21]$ \\
$\mu$ & $0.05[23]$ \\
$f_{c}$ & $2 \mathrm{GHz}$ \\
$L_{\max }$ & 12 \\
$\gamma^{U U}$ & 2 \\
$\mathrm{SNR}$ & $15 \mathrm{~dB}$ \\
$\alpha$ & 1 \\
$\sigma_{n}^{2}$ & $-120 \mathrm{dBm}$ \\
$c$ & $3 \times 10^{8} \mathrm{~m} / \mathrm{s}$ \\
$C_{U G}$ & 3 \\
$L_{\min }$ & 2 \\
$\gamma^{U G}$ & 6 \\
$N$ & 20000 \\
\hline
\end{tabular}

than AF relaying. In this case, $\alpha \geq 1$ can be considered as the penalty to DF relaying. On the other hand, DF can be implemented directly using the traditional transceiver, while AF requires the additional transceiver design, since the relay needs to amplify the received signals. In this case, $0 \leq \alpha \leq 1$ can be considered as the reward to DF relaying. The value of $\alpha$ depends on the energy assumption, network setup, and QoS requirement.

Note that, following (34) and (37), the equalizer coefficients can be calculated using the training symbols. Then, substituting (32) and (24) into (38), the ratio can be rewritten as a function of complexity parameter and training symbols:

$$
\begin{gathered}
\eta\left(\alpha,\left\{x_{k}, \mathbf{y}_{k}^{A}\right\}_{k=1}^{N},\left\{x_{k}, \mathbf{r}_{k}^{D}, \mathbf{y}_{k}^{D}\right\}_{k=1}^{N}\right) \\
=\alpha \frac{\sum_{k=1}^{N} Q\left(\operatorname{sgn}\left(x_{k}\right) \operatorname{Re}\left(\left(\mathbf{w}^{A D}\right)^{H} \mathbf{y}_{k}^{A}+n\right) / \rho_{n}\right)}{\sum_{k=1}^{N} Q\left(\operatorname{sgn}\left(x_{k}\right) \operatorname{Re}\left(\left(\mathbf{w}^{D D}\right)^{H} \mathbf{y}_{k}^{D}+n\right) / \rho_{n}\right)} .
\end{gathered}
$$

Given a complexity parameter $\alpha$ and training symbols, the BER ratio between $\mathrm{AF}$ and $\mathrm{DF}$ with $\mathrm{MBER}$ adaptive equalizers can be calculated.

\section{Numerical Results}

4.1. Simulation Setup. The experimental UAV images, with the size of $1392 \times 1040$ pixels [22], are captured by a mediumaltitude UAV that can cruise at an altitude of $1000 \mathrm{~m}$. The M-UAV plans to transmit the image to GS via R-UAV. The distance between M-UAV and R-UAV and that between R-UAV and GS are both assumed to be $5 \mathrm{~km}$. Antenna heights of UAVs and GS are assumed to be $1000 \mathrm{~m}$ and $10 \mathrm{~m}$, respectively. Thus, the coordinates of the three nodes are $\mathbf{d}=\left(x_{d}, y_{d}, h_{G}\right)=(0,0,10), \mathbf{r}=\left(x_{r}, y_{r}, h_{U}\right)=$ $(5000,0,1000)$, and $\mathbf{s}=\left(x_{s}, y_{s}, h_{U}\right)=(10000,0,1000)$. Simulation parameters, unless explicitly mentioned, are listed in Table 1.

4.2. Results and Discussion. First, we characterize the BER performance of proposed adaptive MBER equalizer against 


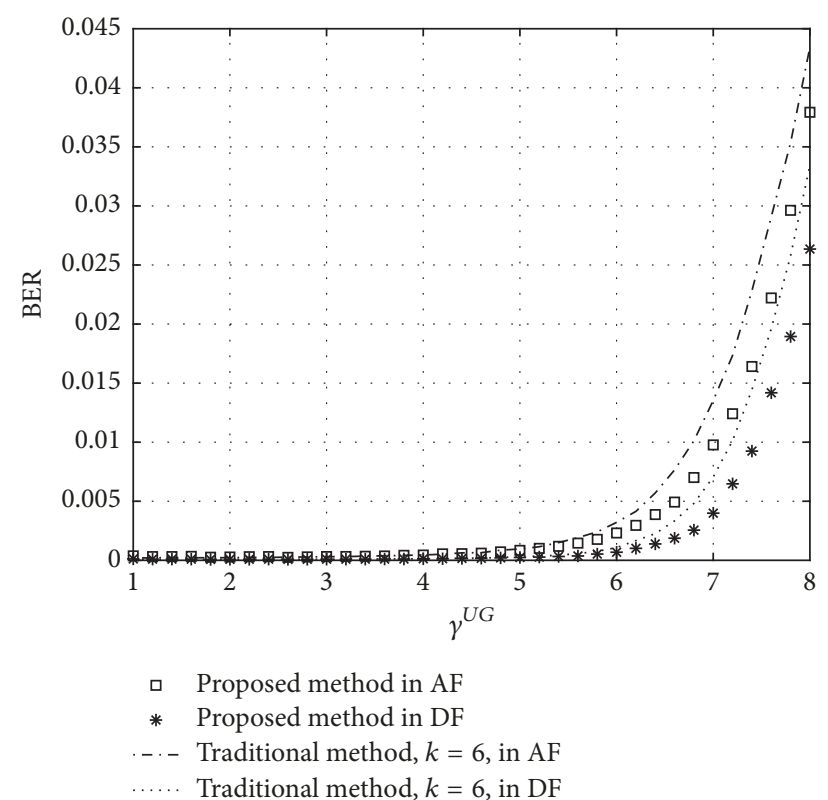

FIGURE 4: BER performance against channel attenuation ratio in UAV-ground channel.

channel attenuation ratio between different multipath components in both UAV-ground and UAV-UAV channels. Considering fixed channel attenuation ratio $\gamma^{U U}=5$ in UAV-UAV channel, Figure 4 shows the BER performance of traditional fixed length and proposed length adaptive equalizers in both AF relaying and DF relaying. It can be seen from this figure that the proposed method has similar BER performance to traditional one in low $\gamma^{U G}$ region but achieves better performance in high $\gamma^{U G}$. The reason is that proposed length adaptive method can adjust the GS' equalizer length according to the channel attenuation ratio of UAV-ground channel. Additionally, BER performance in DF relaying is better than that in AF relaying. Performance comparison with attenuation ratio in UAV-UAV channel is illustrated in Figure 5 with fixed attenuation ratio $\gamma^{U G}=5$ in UAV-ground channel. It can be seen that BER performance of proposed method in DF relaying stays constant, while the performance of that in $\mathrm{AF}$ relaying decreases when $\gamma^{U U}$ increases. This is because length adaptive method can adjust the R-UAV's equalizer length according to $\gamma^{U U}$ in DF relaying, avoiding the drop of BER performance.

Next, we compare the BER performance of AF relaying and DF relaying with the proposed adaptive MBER equalizers against number of training symbols and complexity penalty parameter of DF. BER performance against number of training symbols is shown in Figure 6. Note that these two scenarios should be compared with each other under the same total number of training symbols, resulting in the same end-to-end delay due to training process. Thus, number of training symbols in AF only denotes the symbol number of GS's equalizer. In DF, however, this number denotes the sum of training symbol numbers of R-UAV and GS. Simply assuming that R-UAV and GS in DF relaying use the same



FIGURE 5: BER performance against channel attenuation ratio in UAV-UAV channel.

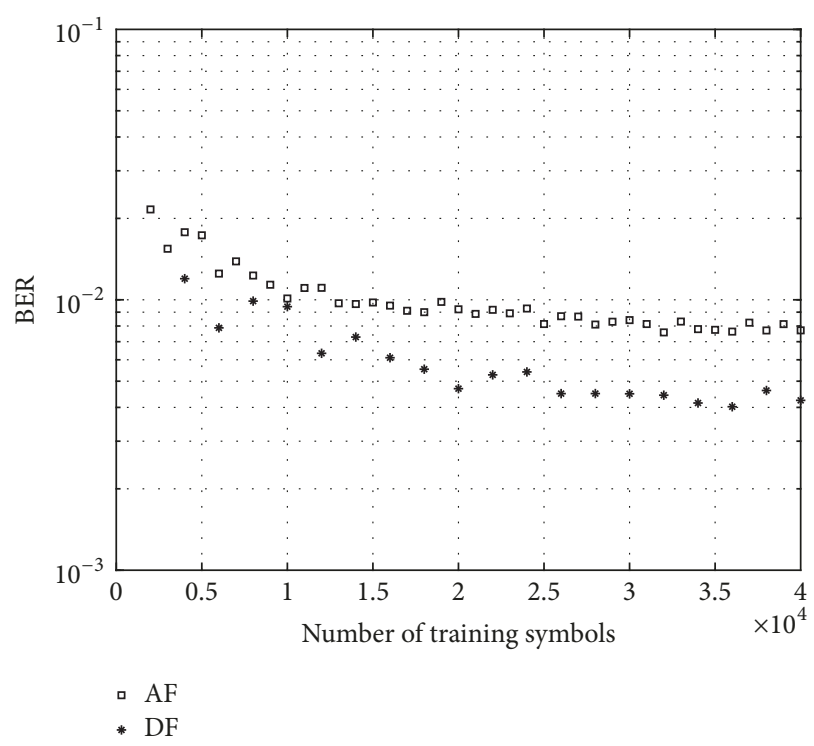

FIGURE 6: BER performance of equalizers against number of training symbols.

amount of training symbols, that is, half of training symbols of GS in AF relaying, DF relaying can still achieve better BER performance than AF relaying but with the slower convergence speed. Figure 7 shows the BER ratio against complexity penalty parameter. It can be seen that DF relaying achieves much better BER performance compared to AF relaying when $\alpha$ is between 0 and 1 and worse BER performance than AF relaying when $\alpha$ is larger than 1 . This phenomenon indicates that AF relaying and DF relaying can be selected based on resource consumption, network setup, and QoS requirement of practical application.

Finally, we examine the quality of received images in both AF relaying and DF relaying with adaptive MBER equalizers 


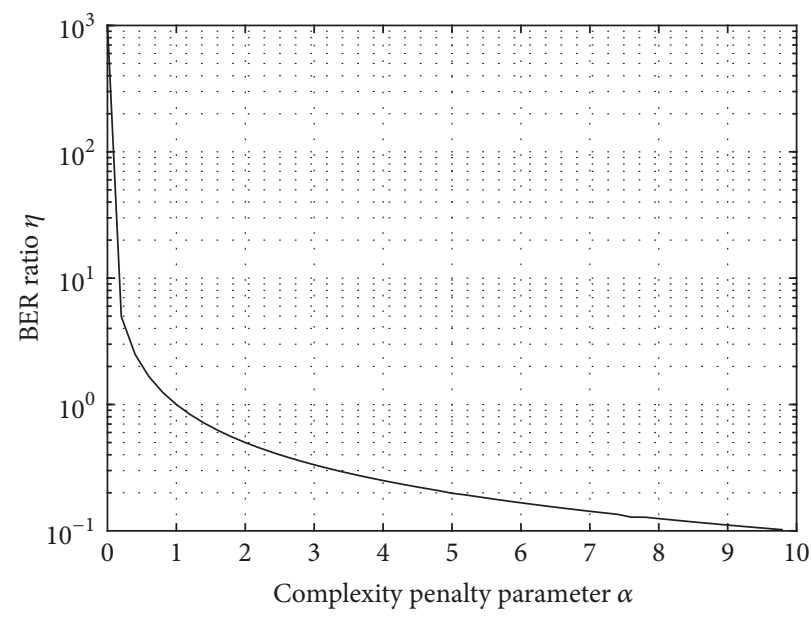

FIGURE 7: BER ratio of AF and DF relaying with adaptive MBER equalizers against complexity penalty parameter.
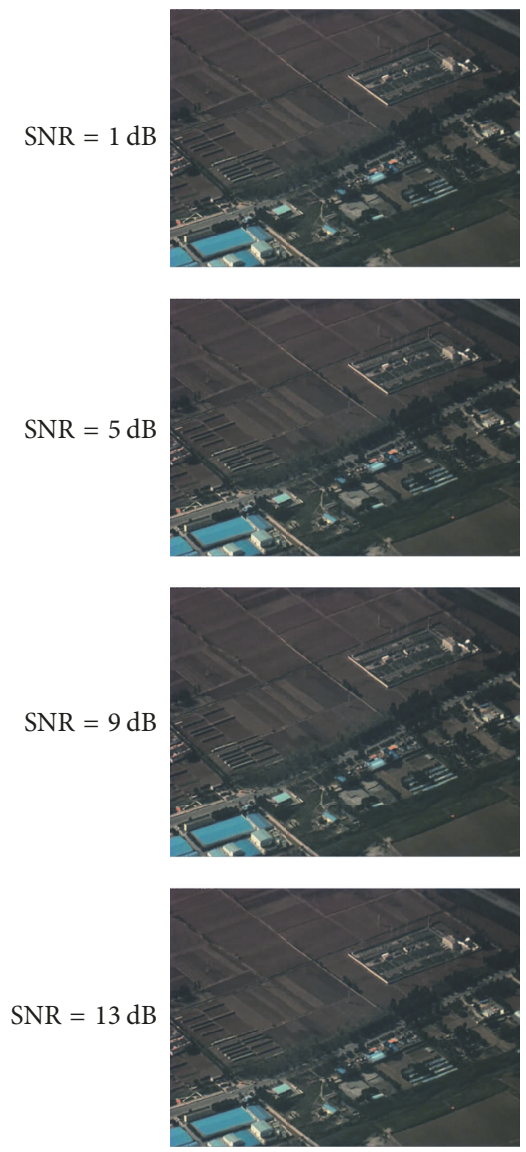

(a)
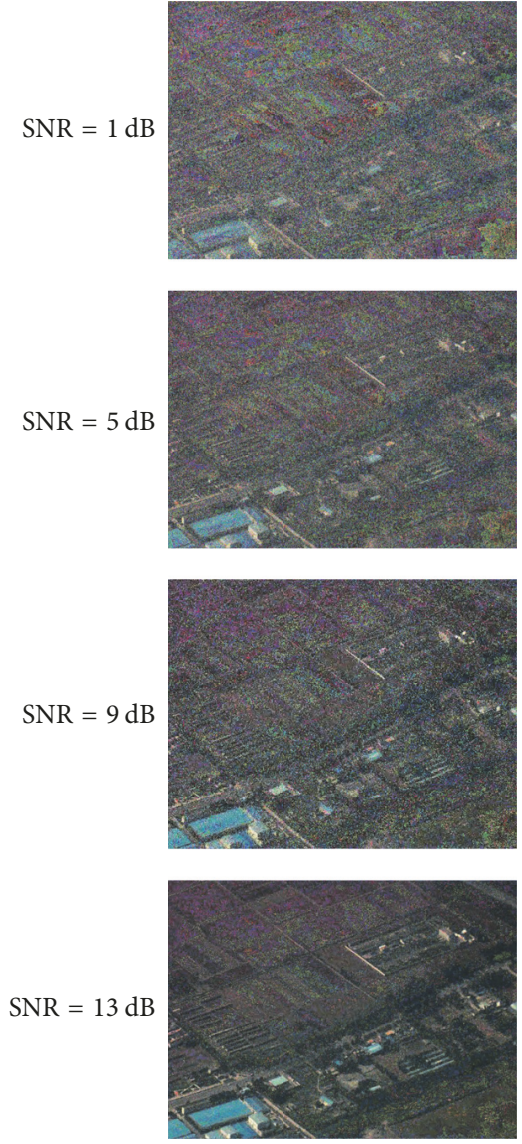

(b)
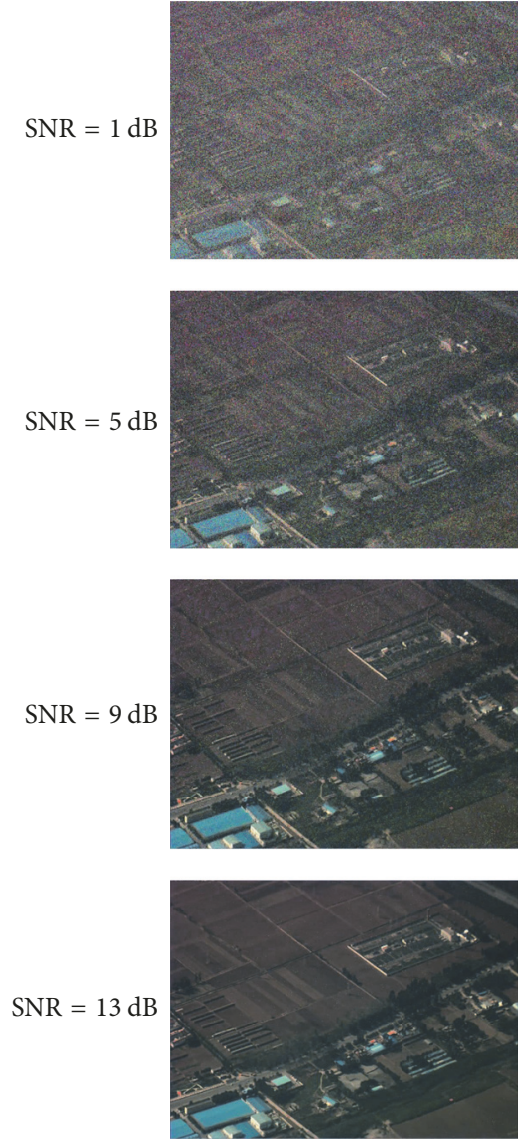

(c)

FIGURE 8: Images in different relaying schemes with adaptive MBER equalizers against SNR: (a) original images at M-UAV, (b) after equalizer in AF relaying at GS, and (c) after equalizer in DF relaying at GS.

against SNR. From Figure 8, we can see that images have very poor quality even after equalizers in the low SNR region. This is because relaying schemes perform poorly in the low SNR region as noted in [21]. Images after equalizers in DF relaying perform better than those in AF relaying at the same SNR.
The average PSNRs of received images at GSs for AF relaying and DF relaying with MBER adaptive equalizers are presented in Figure 9. It is shown that the average PSNR performances in AF relaying and DF relaying schemes increase with SNR. Furthermore, the average PSNR performance in DF relaying 


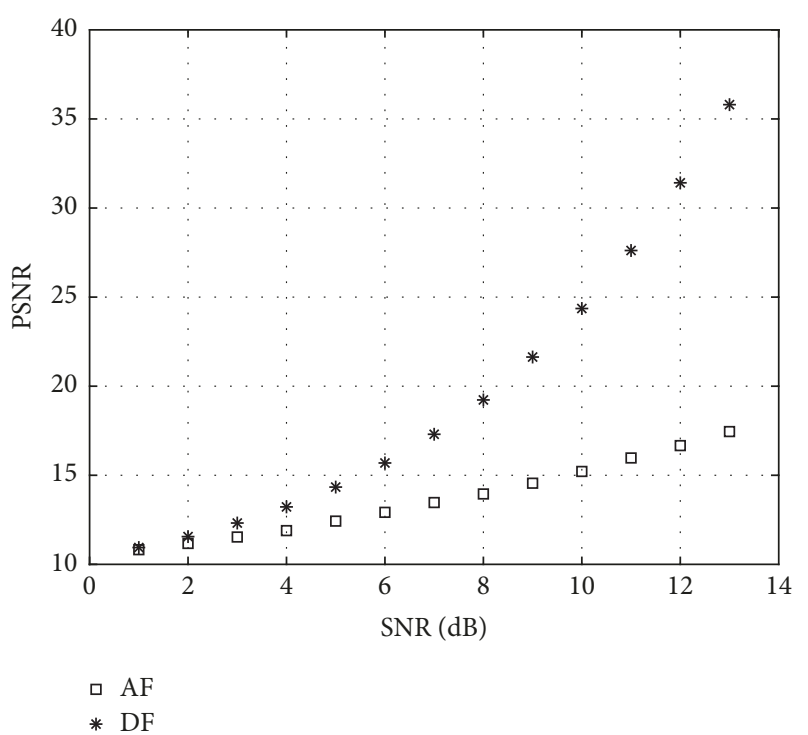

FIGURE 9: Average PSNR performance comparison of received image between AF and DF relaying with adaptive MBER equalizers against SNR.

is better than that in AF relaying across the whole SNR regions and the performance gap significantly increases with SNR.

\section{Conclusion}

In this paper, MBER equalizers with length adaptive method are designed for both AF and DF in UAV-based relaying system. The results show that proposed length adaptive method can achieve better BER performance as channel attenuation ratio between different multipath components increases. Moreover, DF relaying performs better than AF relaying as the channel attenuation ratio in UAV-UAV channel increases. Additionally, results show that the quality of received images is largely improved by proposed adaptive MBER equalizers in both AF relaying and DF relaying.

\section{Conflicts of Interest}

The authors declare that there are no conflicts of interest regarding the publication of this paper.

\section{Acknowledgments}

This work was supported by the National Key Research and Development Program of China (Grant no. 2016YFB0502602) and the National Natural Science Foundation of China (Grant no. 91538204).

\section{References}

[1] J. Kelcey and A. Lucieer, "Sensor correction of a 6-band multispectral imaging sensor for UAV remote sensing," Remote Sensing, vol. 4, no. 5, pp. 1462-1493, 2012.
[2] S. Hayat, E. Yanmaz, and R. Muzaffar, "Survey on Unmanned Aerial Vehicle Networks for Civil Applications: A Communications Viewpoint," IEEE Communications Surveys \& Tutorials, vol. 18, no. 4, pp. 2624-2661, 2016.

[3] G. Tuna, B. Nefzi, and G. Conte, "Unmanned aerial vehicle-aided communications system for disaster recovery," Journal of Network and Computer Applications, vol. 41, no. 1, pp. 27-36, 2014.

[4] Y. Zhou, N. Cheng, N. Lu, and X. S. Shen, "Multi-UAV-Aided Networks: Aerial-Ground Cooperative Vehicular Networking Architecture," IEEE Vehicular Technology Magazine, vol. 10, no. 4, pp. 36-44, 2015.

[5] J. Chen and D. Gesbert, "Optimal positioning of flying relays for wireless networks: A LOS map approach," in Proceedings of the 2017 IEEE International Conference on Communications, ICC 2017, France, May 2017.

[6] A. Al-Hourani, S. Kandeepan, and A. Jamalipour, "Modeling air-to-ground path loss for low altitude platforms in urban environments," in Proceedings of the 2014 IEEE Global Communications Conference, GLOBECOM 2014, pp. 2898-2904, December 2014.

[7] D. W. Matolak and R. Sun, "Air-Ground Channel Characterization for Unmanned Aircraft Systems-Part I: Methods, Measurements, and Models for Over-Water Settings," IEEE Transactions on Vehicular Technology, vol. 66, no. 1, pp. 26-44, 2017.

[8] R. Sun and D. W. Matolak, "Air-Ground Channel Characterization for Unmanned Aircraft Systems Part II: Hilly and Mountainous Settings," IEEE Transactions on Vehicular Technology, vol. 66, no. 3, pp. 1913-1925, 2017.

[9] Y. Zeng, R. Zhang, and T. J. Lim, "Wireless communications with unmanned aerial vehicles: Opportunities and challenges," IEEE Communications Magazine, vol. 54, no. 5, pp. 36-42, 2016.

[10] D. W. Matolak and R. Sun, "Unmanned aircraft systems: Airground channel characterization for future applications," IEEE Vehicular Technology Magazine, vol. 10, no. 2, pp. 79-85, 2015.

[11] D. W. Matolak and R. Sun, "Air-Ground Channel Characterization for Unmanned Aircraft Systems-Part III: The Suburban and Near-Urban Environments," IEEE Transactions on Vehicular Technology, vol. 66, no. 8, pp. 6607-6618, 2017.

[12] M. Rice, M. S. Afran, and M. Saquib, "Equalization in aeronautical telemetry using multiple transmit antennas," IEEE Transactions on Aerospace and Electronic Systems, vol. 51, no. 3, pp. 2148-2165, 2015.

[13] M. Rice, M. Saquib, M. S. Afran, A. Cole-Rhodes, and F. Moazzami, "On the performance of equalization techniques for aeronautical telemetry," in Proceedings of the 33rd Annual IEEE Military Communications Conference, MILCOM 2014, pp. 456461, USA, October 2014.

[14] Q. Z. Ahmed, M. Alouini, and S. Aissa, "Bit error-rate minimizing detector for amplify-and-forward relaying systems using generalized gaussian kernel," IEEE Signal Processing Letters, vol. 20, no. 1, pp. 55-58, 2013.

[15] Q. Z. Ahmed, S. Ahmed, M.-S. Alouini, and S. Aïssa, "Minimizing the symbol-error-rate for amplify-and-forward relaying systems using evolutionary algorithms," IEEE Transactions on Communications, vol. 63, no. 2, pp. 390-400, 2015.

[16] Q. Z. Ahmed, K.-H. Park, M.-S. Alouini, and S. Aissa, “Optimal linear detectors for nonorthogonal amplify-and-forward protocol," in Proceedings of the 2013 IEEE International Conference on Communications, ICC 2013, pp. 4829-4833, Hungary, June 2013. 
[17] P. G. Sherwood, X. Tian, and K. Zeger, "Channel code blocklength and rate optimization for progressive image transmission," in Proceedings of the 1st IEEE Annual Wireless Communications and Networking Conference, WCNC 1999, pp. 978-982, USA, September 1999.

[18] A. K. Dutta, K. V. S. Hari, and L. Hanzo, "Linear transceiver design for an amplify-and-forward relay based on the MBER criterion," IEEE Transactions on Communications, vol. 62, no. 11, pp. 3765-3777, 2014.

[19] D. Jordane, "Electromagnetic waves and radiating systems. lem plus $0.5 \mathrm{em}$ minus $0.4 \mathrm{em}$ Prentice-Hall Of Lndia Private Limited," Electromagnetic waves and radiating systems. 1em plus 0.5em minus 0.4em Prentice-Hall Of Lndia Private Limited, 1967.

[20] M. Rice, T. Nelson, J. Palmer, C. Lavin, and K. Temple, "SpaceTime Coding for Aeronautical Telemetry: Part II - Decoder and System Performance," IEEE Transactions on Aerospace and Electronic Systems, vol. 53, no. 4, pp. 1732-1754, 2017.

[21] S. Chen, S. Tan, L. Xu, and L. Hanzo, "Adaptive minimum errorrate filtering design: A review," Signal Processing, vol. 88, no. 7, pp. 1671-1697, 2008.

[22] H. Li, X. Li, W. Ding, and Y. Huang, "Metadata-assisted global motion estimation for medium-altitude unmanned aerial vehicle video applications," Remote Sensing, vol. 7, no. 10, pp. 1260612634, 2015.

[23] S. Chen, "Adaptive minimum bit-error-rate filtering," IEE Proceedings Vision, Image and Signal Processing, vol. 151, no. 1, pp. 76-85, 2004. 


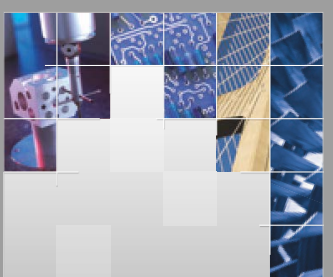

\section{Enfincering}
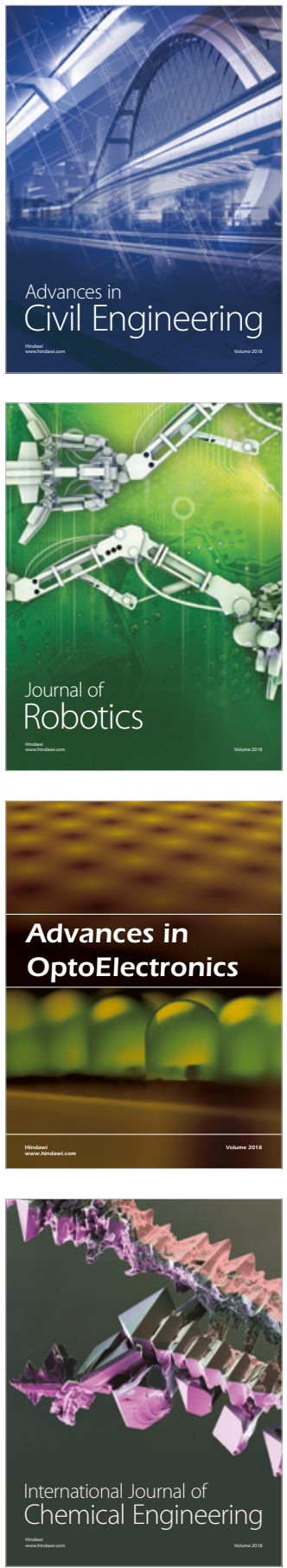

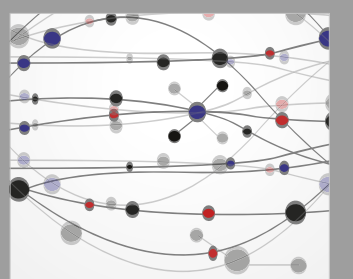

\section{Rotating \\ Machinery}

The Scientific World Journal

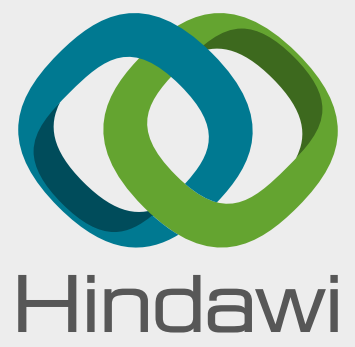

Submit your manuscripts at

www.hindawi.com
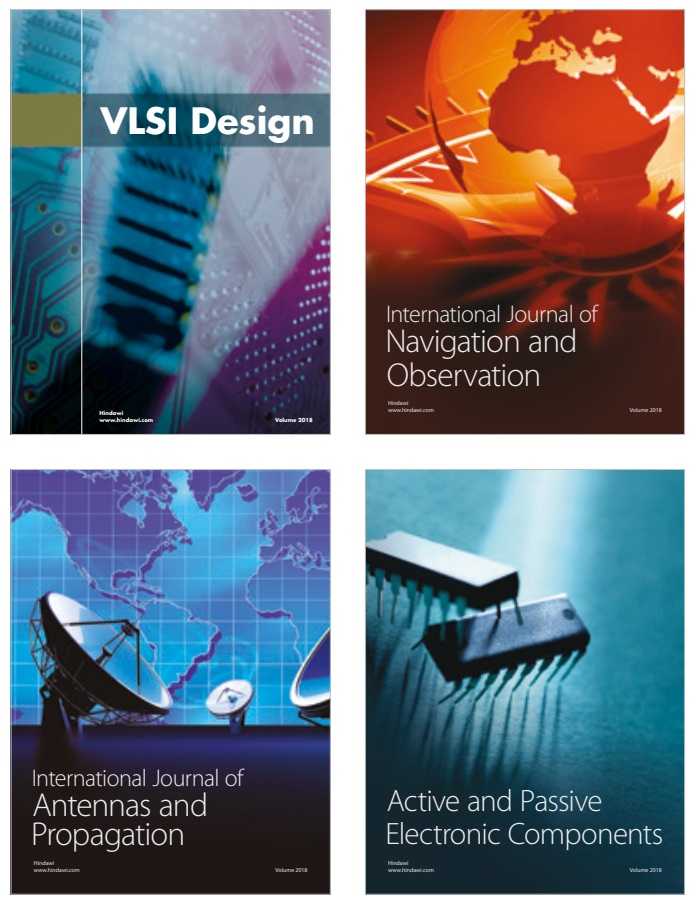
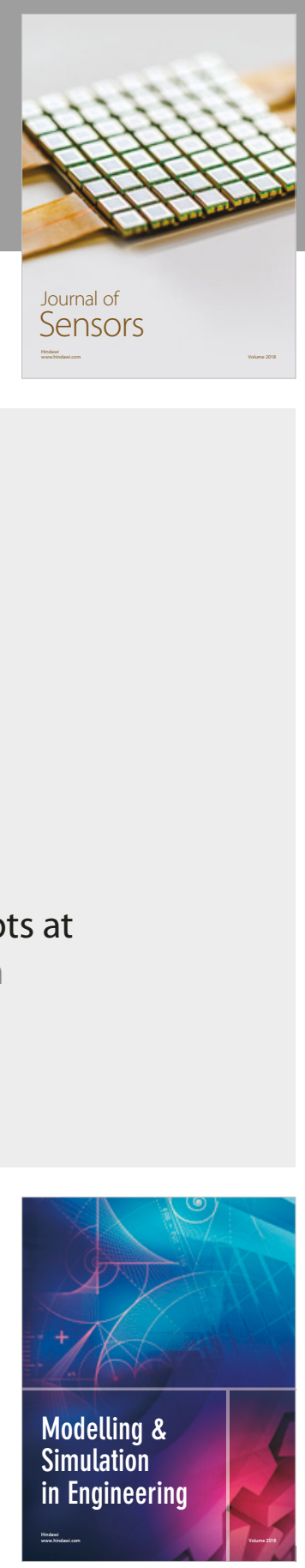

\section{Advances \\ Multimedia}
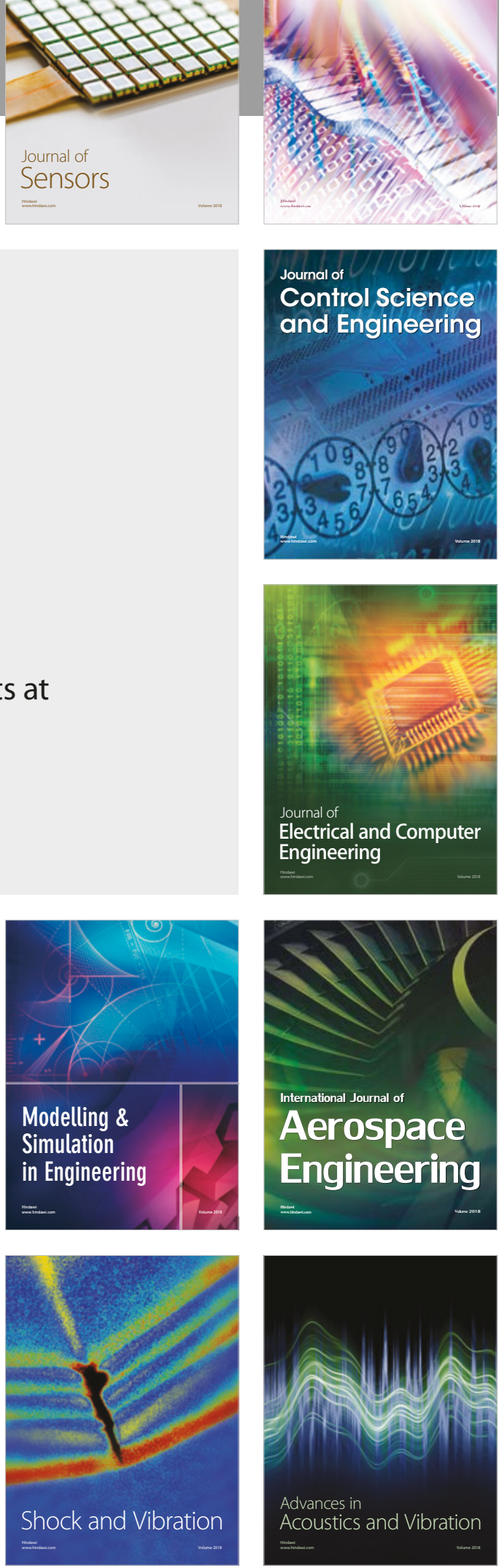